\title{
Efficacy of hydrotherapy treatment for the management of chronic low back pain
}

Mirmoezzi, Masoud ; Irandoust, Khadijeh ; H’mida, Cyrine ; Taheri, Morteza ; Trabelsi, Khaled ; Ammar, Achraf ; Paryab, Nesa ; Nikolaidis, Pantelis T ; Knechtle, Beat ; Chtourou, Hamdi

\begin{abstract}
Aims The study investigated the influence of hydrotherapy method-based McKenzie and Williams among patients with non-specific low back pain (NSLBP). Methods Semi-experimental pretest-posttest with a control group design trial was conducted at Health Service Center, Qazvin, Iran. Twenty-eight NSLBP patients (16 men and 12 women, age: $42.5 \pm 7$ years) were recruited. Hydrotherapy program developed based on McKenzie and Williams therapy was performed individually 3 days per week for 20 sessions. Therefore, the study compared a hydrotherapy group (based on McKenzie and Williams therapy) to a control group. Participants attended a hydrotherapy program under the supervision of a hydrotherapist. The Numeric Pain Rating Scale (NPRS), RolandMorris Disability Questionnaire (RMDQ), and Straight Leg Raise Test (SLRT) were measured at the beginning, middle, and at the end of the 20 sessions to determine pain and functionality of the patient's improvement. Results Overall, NPRS, RMDQ, and SLRT scores were improved in hydrotherapy group in the 10th session and 20 th session compared with baseline $(\mathrm{p} \leq 0.001)$ and control group $(\mathrm{p} \leq 0.001)$. No difference in the treatment variables between the 10 th session and the 20 th session was observed $(p>0.05)$. Conclusions The results indicated that the NSLBP symptom was improved after 10 sessions of hydrotherapy program developed based on McKenzie and Williams therapy.
\end{abstract}

DOI: https://doi.org/10.1007/s11845-020-02447-5

Posted at the Zurich Open Repository and Archive, University of Zurich

ZORA URL: https://doi.org/10.5167/uzh-196854

Journal Article

Accepted Version

Originally published at:

Mirmoezzi, Masoud; Irandoust, Khadijeh; H’mida, Cyrine; Taheri, Morteza; Trabelsi, Khaled; Ammar, Achraf; Paryab, Nesa; Nikolaidis, Pantelis T; Knechtle, Beat; Chtourou, Hamdi (2021). Efficacy of hydrotherapy treatment for the management of chronic low back pain. Irish journal of medical science, 190(4):1413-1421.

DOI: https://doi.org/10.1007/s11845-020-02447-5 
Efficacy of hydrotherapy treatment for the management of chronic

Masoud Mirmoezzi: massoudmirmoezi@live.com

Khadijah Irandoust: irandoust@soc.ikiu.ac.ir

Cyrine H'mida: sirinehmida@hotmail.fr

Morteza Taheri: intl.office@pst.ikiu.ac.ir

Khaled Trabelsi: trabelsikhaled@gmail.com

Achraf Ammar: ammar.achraf@ymail.com

Nesa Paryab: nesashooter@yahoo.com

Pantelis T. Nikolaidis: pademil@hotmail.com

Beat Knechtle: beat.knechtle@hispeed.ch

Hamdi Chtourou: h_chtourou@yahoo.fr 


\section{ABSTRACT}

44 Aims: The study investigated the influence of hydrotherapy method-based McKenzie and Williams

45 among patients with non-specific low back pain (NSLBP). Methods: Semi experimental pretest-

46 posttest with a control group design trial was conducted at Health Service Center, Qazvin, Iran.

47 Twenty-eight NSLBP patients (16 men and 12 women, age: $42.5 \pm 7 \mathrm{yrs}$ ) were recruited.

48 Hydrotherapy program developed based on McKenzie and Williams therapy was performed

49 individually three days per week for 20 sessions. Therefore, the study compared a hydrotherapy

50 group (based on McKenzie and Williams therapy) to a control group. Participants attended a

51 hydrotherapy program under the supervision of a hydrotherapist. Numeric Pain Rating Scale

52 (NPRS), Roland-Morris Disability Questionnaire (RMDQ) and Straight Leg Raise Test (SLRT)

53 were measured at the beginning, middle and at the end of the 20 sessions to determine pain and

54 functional of the patient's improvement. Results: Overall, NPRS, RMDQ and SLRT scores were

55 improved in hydrotherapy group in $10^{\text {th }}$ session and $20^{\text {th }}$ session compared with baseline $(\mathrm{p} \leq 0.001)$

56 and control group $(\mathrm{p} \leq 0.001)$. No difference in the treatment variables between the $10^{\text {th }}$ session and

57 the $20^{\text {th }}$ session was observed $(\mathrm{p}>0.05)$. Conclusions: The results indicated that the NSLBP

58 symptom was improved after 10 sessions of hydrotherapy program developed based on McKenzie

59 and Williams therapy.

60

61 Keywords: Pain; Therapy; Disability; Health.

62 


\section{INTRODUCTION}

Low back pain (LBP) is one of the most common conditions that impair individuals'

65 functional capacity in activities of daily living and at work, as well as their general health and

66 quality of life [1]. LBP is also responsible for significant part of the demand for health services and

67 is a multidimensional phenomenon involving psychosocial, behavioral and pathophysiological

68 processes [2]. So, since it is a disabling condition with high costs both for individuals and the

69 society, it represents an important global and socioeconomic problem [3]. Many patients also want

70 faster return of functional abilities and reduced pain for economic reasons [4].

71 Many therapies have been used for the treatment of LBP such as drug therapy, laser therapy,

72 ozone therapy, dry-land exercise, ultrasound, chiropractic, hydrotherapy and finally surgery [5]; but

73 the objectives are the same: pain control, disability prevention and resuming of suppressed daily

74 activities and of work [6].

Hydrotherapy an increasingly popular form of physiotherapy treatment for a large variety of

conditions likes subacute and chronic LBP. Hydrotherapy is defined as a pool therapy program

designed for individuals to improve neuromuscular and musculoskeletal functions, which is

conducted and supervised by qualified personnel ideally in a specifically built hydrotherapy pool

[7]. Effects of unique properties of the water (buoyancy, resistance, flow and turbulence) allow the

80

performance of movements which are normally difficult or impossible on land $[4,8]$.

Applying an appropriate treatment is important given the duration of flares and the relapses

rate. Therefore, this study will explore the two most widespreaded methods, Williams and

McKenzie which is simulated in water. Mackenzie and Williams are two methods of exercise

therapy that have a favorable effect on some passive therapy methods. Because it is unpleasant due

to training in therapeutic or sports treatment environments as well as dry-land exercises, some of

which have muscle weakness and have to perform therapeutic exercise in the face of gravity. For this reason, we proposed a simulated method in water $[9,10]$. Since aquatic therapy is safe, welltolerated, and an enjoyable form of exercise produced improvement in disability and quality of life 
89 of the patients with LBP, the aim of this study is to evaluate the efficacy of hydrotherapy method-

90 based McKenzie and Williams on chronic back pain by conducting a randomized controlled trial.

91 Accordingly, we hypothesized that 20 sessions of hydrotherapy protocol has a positive effect on

92 pain intensity scale and disability in patients with NSLBP.

93

94

95

96

97

98

99

100

101

102

103

104

105

106

107

108

109

110

111

112

113 


\section{METHODS}

\section{Ethical approval}

117 The research was approved by local ethical committee of Imam Khomeini International

118 University (ref. no: 11596).

Participants

This study was a semi experimental pretest-posttest with a control group. Twenty-eight patients suffering from subacute and chronic non-specific LBP (16 men and 12 women, age:

familiar with either McKenzie's or William's exercises, volunteered to participate in this study. The sample size was estimated based on G power software (G-Power for Windows, Version 3.1,

126 Duesseldorf, Heinrich-Heine University) with a statistical power of 95\%, effect size of 0.5 and 127 alpha of $0.05,26$ subjects estimated [11]. To compare an hydrotherapy group (based on McKenzie

\section{8 and Williams therapy) to a control group, the participants were divided into two equal groups by}

129 Covariate-Adaptive Randomization (CAR) procedure based on the variable Numeric Pain Rating

130 Scale (NPRS) of patients in initial evaluation. For observing the ethics, besides getting consent from

131 all participants, it was explained that the results of the study were purely for research purposes and

132 would be published collectively without mentioning the names of the individuals. Their

133 participation in the study was also optional, and they could leave it at any stage.

134 The inclusion criteria included all patients with age 18-60 years with NSLBP for more than 135 one year. The patients experience LBP for at least the previous 3 months; Roland-Morris Disability

136 Questionnaire (RMDQ) score was 4 or more in patients. They performed not regularly physical

137 exercises. MRI of lumbar spine were performed and evaluated before inclusion by radiology

138 specialists. Disk dehydration, bulging, protrusion, extrusion and degeneration were cases involved

139 in the study. Patients agreed to avoid physical treatments, other than trial treatments, for two 
months. They did not have histories of rheumatic, neurological and congenital diseases, cognitive

141 and nervous systems disorders, waist and hip fractures or dislocations, sacroiliac joint dysfunctions,

142 malignancy on vertebrae and lumbar spinal stenosis. All potential participants underwent Mini

143 Mental State Examination (MMSE) and those with a score of less than 24 were excluded [12].

144 Patients with increased back pain and inability to continue treatment were excluded from the

145 research.

147 Procedure

148 Patients, upon entering the study, were evaluated by NPRS, RMDQ and Straight Leg Raise

149 Test (SLRT) as common Provocative test. Investigators for each questionnaire and test were blinded 150 to allocations of interventions.

151 NPRS is a rating scale $0-10$ in which 0 means no pain and 10 means the worst pain ever 152 experienced. Patients had their pain assessed and allocated a number from zero to ten to them [13].

153 The RMDQ-24 is most sensitive for patients with mild to moderate disability due to acute,

154 sub-acute or chronic low back pain. RMDQ is a self-administered disability measure in which 155 greater levels of disability are reflected by higher numbers on a 24-points scale [12].

156 Passive SLRT is one of the most commonly performed tests in clinical practice. To conduct

157 this test, have the pateint lay supine and passively elevate the fully extended leg of the affected side

158 to 30-70 degrees. The measurements were taken by the freeware motion analysis software Kinovea

159 (version 0.8.15, available for download at: http://www.kinovea.org) and and Lumix digital camera

160 (Panasonic Corporation, Osaka, Japan). The reflective markers were attached on the major

161 anatomical landmarks. At the moment the participant expressed the perception of LBP or leg pain,

162 he raises the hand to camera and the angle between two assumptive lines between greater trochanter

163 to the lateral malleolus and greater trochanter to the internal malleolus was measured [10].

164 Patients were randomly divided into treatment and control groups and were evaluated in three

165 stages: initial (one day before first session), middle (10 ${ }^{\text {th }}$ session) and final $\left(20^{\text {th }}\right.$ session). 
166 Hydrotherapy sessions were conducted 3 days/week for 20 sessions based on Table 1. These

167 exercises are designed based on the McKenzie and Williams methods and the exercises of the

168 Aquatic Exercise Association (AEA) recommendation [14]. Exercises in water were administered in

169 the hydrotherapy pool unit (water temperature range $32^{\circ} \mathrm{C}-34^{\circ} \mathrm{C}$ ) by the researchers with the

170 assistance of an experienced hydrotherapist (the hydrotherapist was one of the instructors and

171 coaches of the Iranian Sports Medicine Federation) in aquatic exercise protocols.

172 Following a 1-week washout period (during the washout, the patients stopped any

173 nonsteroidal anti-inflammatory drugs or any other analgesic drugsthat used previously), the patients

174 were invited to the therapy sessions. Questionnaires consent and tests were obtained from all

175 patients one day before exercise (baseline). Specific exercises were prescribed for hydrotherapy

176 group using classical approach of warm-up, exercise-phase and cool-down principles. Warm-up and

177 cood down phases comprised 5 min of high spot stepping 2 widths of pool walking forward,

178 backward and sideways for hydrotherapy group [14]. The control group was followed up for a

179 period of 20 sessions. All participants had no occupational activity during the 20 sessions of the

180 treatment and were told to not perform bad daily habits in sleeping, sitting and standing.

181 Participants in hydrotherapy group follow the two hours pain rule. If pain was higher than

182 normal two hours after exercising (might indicate overexertion), the exercise should be less intense

183 in the next session according to the AEA instructions [14,15]. Also, all functional exercises were

184 performed in the ROM up to pain threshold and when the sensation pain started, one would either

185 stop exercising or increase ROM.

186 Hydrotherapy method-based McKenzie and Williams

187 In the Williams's method, the purpose was to reduce pain and to ensure stability of the lower

188 trunk by toning the abdominal muscles, buttocks and hamstrings, altogether with the passive extent

189 of hip flexors and sacrospinalis muscles [10]. A vicious circle of mechanical overloading, muscle

190 contractions of the abdominals and of the iliopsoas, catabolic cell response, and degeneration of the

191 water-binding extracellular matrix leading to an increase nerve root irritation in the lumbosacral 
192 area [16]. Supine position and implementation of the Williams's protocol in stage 1 leading to

193 relaxation of the paravertebral muscles and help to the inhibition phenomenon. In stage 2, vertical

194 positions allow the patient to make transition painlessly with gradual loading. Finally stage 3,

195 toning the abdominal muscles and back extensors, in orthostatism, is to achieve a neutral position of

196 the pelvis and to create an abdominal pressure capable of taking some of the pressure placed on the

197 lower lumbar intervertebral discs [17].

198 In the McKenzie's method, the purpose was based on lumbar extension exercises, and on

199 lumbar flexion ones. These are not fixed or divided into different stages, but comply with the

200 principle of progressivity, and their aim is centralizing pain, pain relief and regaining functionality.

201 McKenzie believes that pain occurrence is natural because the nucleus pulposus edges are highly

202 innervated and pressure acts like a mechanical aggresion on the annulus $[9,3]$.

203 The hydrotherapy program designed based on the Williams and McKenzie mechanisms involves the

204 following stages: Stage (1) includes horizontal floating and relaxation, which relieves excess

205 pressure on the lumbar discs, and water flow and turbulence also helps the patient to recover

206 mentally and physically [8]. Stage (2) includes vertical floating with/without the weight cuffs are

207 attached to the ankles which causes release to the vertebral discs and proper spacing between the

208 vertebrae and reduced pressure on the nerve roots [6]. Stage (3) includes release the intervertebral

209 discs in four anterior, posterior, and lateral directions that place the nucleus pulposus in position and

210 the pressure is removed from the fibrous layers and helps to feed the disc and to restore its height by

211 absorbing unauthenticated liquid can withstand compressive forces [10]. Stage (4) includes

212 strengthening muscles, especially in the body's anti-gravity muscles to maintain body structure in a

213 static and dynamic posture [15]. Stage (5) includes flexibility and mobility in different directions to

214 affect the body's ability to function properly [4].

215

216

217 
Statistical analysis was performed using SPSS (SPSS for Windows, Version 19.0, Chicago,

220 SPSS Inc, UnitedStates). Normality of the data was examined with Shapiro-Wilk test ( $\mathrm{p}>0.05)$.

221 Nonparametric methods were applied when data fell outside the normal distribution. Two-way 222 repeated measures ANOVA were used to measure a dependent variable over three time periods

223 (before the $1^{\text {st }}$ session, after the $10^{\text {th }}$ session and after the $20^{\text {th }}$ session) between hydrotherapy and

224 control groups (SLRT). Nonparametric tests (Friedman, Wilcoxon signed-rank and Mann-Whitney

$225 \mathrm{U}$ tests) were used to determine whether differences existed among the over three time points and

226 groups for categorical baseline variables and the study outcome measures. Friedman's test with

227 Wilcoxon signed-ranks tests as a post-hoc test was applied to assess inter-group differences and

228 Mann-Whitney U-tests were then used to examine intra-group differences for nonparametric data

229 (NPRS, RMDQ). Statistical significance was set at $\mathrm{p}<0.05$ and Bonferroni correction was applied to

230 allow for multiple comparisons (Figure 1). 


\section{RESULTS}

245 Demographic data were compared between the control group and the LBP group using

246 independent t-tests and chi-square tests for scale data (e.g., age, body weight) and for nominal data

247 (gender, NPRS, RMDQ), respectively (Table 2).

248 Each pre-measure of groups was analyzed by Mann-Whitney U test which demonstrated no

249 significant differences between the groups with respect to NPRS ( $\mathrm{P}=0.251)$, and $\mathrm{RMDQ}(\mathrm{P}=0.644)$.

250 Pre-measure of groups was analyzed by independent t-test which demonstrated no significant

251 differences between the groups for SLRT $(\mathrm{P}=0.527)$.

252 The result of the analysis on periods (before the $1^{\text {st }}$ session, after the $10^{\text {th }}$ session and after the $20^{\text {th }}$

253 session) of NPRS and RMDQ in both groups by Friedman's test with Wilcoxon signed-ranks tests

254 as a post-hoc test indicated that the hydrotherapy group have showed a significant difference in the

255 time series $\left(\chi^{2}(2)=24.81, p<0.001\right.$; Table 2$)$ and hydrotherapy group have a positive effect on

256 NPRS and RMDQ in $10^{\text {th }} \operatorname{session}(Z=-3.32, p=0.001)$ and $20^{\text {th }} \operatorname{session}(Z=-3.22, p=0.001)$ with

257 baseline (Figure 2). The Mann-Whitney U test showed that hydrotherapy exercises in $10^{\text {th }}$ session

258 and $20^{\text {th }}$ session were significantly more effective than control group. Means and standard

259 deviations of the measured variable are also shown in Table 3.

260 As shown in Table 3, the results of two-way repeated measures ANOVA for SLRT show

261 that there is a significant difference within-group $\left(\mathrm{F}_{2,13}=8.18, \mathrm{p}<0.001\right)$ and there is a significant

262 difference between groups $\left(\mathrm{F}_{1,26}=9.87, \mathrm{p}<0.015\right)$. There is no significant difference between

263 interactions of groups and session. A Bonferroni post hoc test showed that hydrotherapy group has

264 higher SLRT in $10^{\text {th }}$ session $(\mathrm{p}<0.001)$ and $20^{\text {th }}$ session $(\mathrm{p}<0.001)$ than baseline. Also,

265 hydrotherapy exercises in $10^{\text {th }}$ session and $20^{\text {th }}$ session were significantly more effective on SLRT

266 than control group ( $p<0.001$; Figure 2). 
269 With regard to the results of this study, the hydrotherapy protocol has a positive effect on self-

270 administered pain intensity scale and disability based on health status measure in patients with

271 chronic LBP after $10^{\text {th }}$ and $20^{\text {th }}$ sessions compared to baseline. Also, this protocol can increase the

272 range of motion (ROM) in SLRT for decrease nerve root irritation in the lumbosacral area. Based

273 on our results, there was no significant difference between the $10^{\text {th }}$ and the $20^{\text {th }}$ sessions of the

274 treatment variables.

We hypothesized that, the hydrotherapy protocol has a positive effect on pain intensity scale and disability in patients with NSLBP after the $10^{\text {th }}$ and $20^{\text {th }}$ sessions and based on the findings, this hypothesis was confirmed. We also hypothesized that continuing the training teatment for $20^{\text {th }}$ sessions has a greater impact on reducing the patient's LBP; however, based on the findings, this hypothesis was not confirmed and no difference was found between $10^{\text {th }}$ and $20^{\text {th }}$ sessions of the treatment variables.

Based on our findings, hydrotherapy shows significant positive changes in the pain intensity, 282 disability in physical functioning and reduce nerve root irritation in LBP after 10 sessions which 283 affirms the findings of some meta analyses on exercise therapy and therapeutic aquatic exercise in 284 management and treatment of LBP $[18,19]$. Authors suggested that exercise therapy and therapeutic 285 aquatic exercise, including abdominal stabilization exercise, weight relief treatment, flexibility exercise seems to be slightly effective at pain reduction and functional improvement for chronic LBP [18].

Water immersion decreases axial loading of the spine and, through the effects of buoyancy, 289 allows the realisation of movements that are normally difficult or impossible on land [19].

290 Buoyancy reduces stress on joints, muscles and enables greater range of movement via supporting 291 the weight of the body and warm water increases muscle efficiency $[14,20]$. The hydrostatic 292 pressure and depth allow the patients to perform exercise more easily. Water viscosity and surface 293 tension creates resistance to all active exercises and strengthen the muscles [21]. A desired exercise 
294 intensity can be achieved by adjusting the velocity of movement in the water [22]. Water-based

295 physical activity enhances balance and coordination while stimulating, visual, vestibular, and

296 perceptual systems [14]. By utilizing the unique properties of water (buoyancy, resistance, flow and

297 turbulence) a graded exercise programme from assisted to resisted movements can be created to suit

298 the patients' needs and function [19].

299 Pain has profound and wide-ranging effects on motorfunction. People who experience

300 chronic pain have altered movement patterns [23]. Patients with chronic LBP that have lower

301 physical activity levels had higher levels of disability [24]. LBP and disability affect the reduction

302 of ROM in hip flexion. SLR test is neurodynamic tests that check the mechanical movement of the

303 neurological tissues as well as their sensitivity to mechanical stress or compression in lumbosacral

304 nerve root irritation. Abnormal neurodynamic responses and consequently symptoms in patients

305 with chronic radiculopathy may be due to a pathomechanic problem [25]. It sounds that water

306 exercises enhance ROM and function of the core stabilizer muscles by increasing neuromuscular

307 coordination of agonist and antagonist muscles [15]. Sensory input from water pressure and

308 temperature may decrease feelings of pain [22]. Exercise in water takes the weight off a painful

309 joint while also providing greater flexibility and range of motion of the spine, enhanced and reduces

310 stress on the back pain and also, neutralization of the gravity pressure by floating during this

311 stretching, lumbar intradiscal pressure decreases [7].

312 In addition to the water features mentioned sufficiently in the literature, hydrotherapy program that

313 had a positive effect on pain and disability reduction during 10 training sessions is probably due to

314 the following reasons:

315 The patient's horizontal and vertical floating relaxes and reduces overload, discharging the

316 weight of the patient's torso resulting in the body being in the postural correction, the body

317 alignment along the line of gravity and maintaining posture and the neutral spine position and

318 relaxation of vertebral muscles. Next, attaching a weight cuff to the patient's ankle and noodle in the

319 arms creates a good stretch over the spine, which reduces disc pressure on the thecal sac, nerve 
320 roots and creates the needed intervertebral distance. Isotonic movements with the weight cuff help

321 further this release in addition to strengthening the body's core muscles. In the later stages, the

322 patient exercises in various directions (flexion, hyperextension and lateral flexion) due to the release

323 of the disc, the strengthening of the muscles, especially core muscles, and enhanced balance and

324 coordination of muscle groups to stabilize the spine, spinal joint mobilization, deep tissue

325 mobilization and finally movement of the nucleus pulposus towards the central region. And it's,

326 also, called neurodynamic treatments for LBP.

327 Possible reasons for the lack of significant effects on the pain intensity and disability in the

328 second 10 sessions may be the type of exercises and the focus on these exercises on muscle

329 strength, flexibility and mobility, which emphasizes maintaining the desired condition. Although

330 this difference was not significant, it seems that the 10 sessions training program only helps to

331 reduce the pain intensity and disability effects, and the follow-up sessions help to adjust and

332 maintain the patient's good posture along with low pain and disability and there is a need to

333 continue the practice. Probably the reason for the slight improvement in LBP symptoms in the

334 control group seems to be due to resting, decrese daily activity, and stop daily bad habits in paients,

335 that's the first step in the treatment of LBP.

336 Some limitations of the present study should be highlighted. The duration of hydrotherapy's

337 exercises was short. Long-term changes due to this protocol were not examined. Our sample size

338 was relatively small to detect significant improvements in outcomes. Patients were not blinded,

339 although investigators were blinded. All participants may be somewhat interested in hydrotherapy,

340 so they would expect positive effects of hydrotherapy and report less pain intensity and disability.

341 The tests are self-administered measurement; more accurate tests such as the EMG registration may

342 be more appropriate for patients. No socio-demographic and psychological factors measurement

343 that potentially impacts on the results such as educational background, occupation or

344 socioeconomic status, fear, anxiety, stress, depression and lower self-esteem were investigated. 
345 Also, nutrition habits were not assessed in the present study. Further fully powered studies were

346 required to investigate efficacy and its validity.

$347 \quad$ Conclusions

348 Symptom of NSLBP improved by hydrotherapy program developed based on McKenzie and

349 Williams therapy in 10 sessions. In the second 10 sessions of the training program, which focused

350 on strength and mobility, the exercises only help to stabilize the patients' condition. According to

351 the present study, hydrotherapy is suggested for patients suffering from chronic LBP. Hydrotherapy

352 method-based McKenzie and Williams can be a safe and effective method for patients with chronic

353 LBP. Therapists can use this method for specific groups and according to the patient's needs.

354

355

356

357

358

359

360

361

362

363

364

365

366

367

368

369

370 


\section{Authors' contribution:}

372 Conceptualization, methodology and data collection: Masoud Mirmoezzi, Khadijah Irandoust,

373 Morteza Taheri

374 Writing — original draft preparation: Masoud Mirmoezzi, Khadijah Irandoust, Cyrine H'mida,

375 Morteza Taheri

376 Writing - review and editing: Khaled Trabelsi, Nesa Paryab, Achraf Ammar, Pantelis T. Nikolaidis,

377 Beat Knechtle, Hamdi Chtourou

378

379 Statement

380 All authors read and approved the final version of the manuscript.

381

382 Conflict of interest

383 No conflict of interest

384

385 Funding

386 No funding

387

388

Acknowledgment

389 The authors would like to thank Mr. M. Shakibzadeh for drawing the hydrotherapy images and all

390 the patients who participated in this study.

391

392

393

394

395

396 
398 1. Irandoust K, Taheri M (2015) The effects of aquatic exercise on body composition and 399 nonspecific low back pain in elderly males. Journal of physical therapy science 27 (2):433-435.

$400 \quad$ doi:10.1589/jpts.27.433

401 2. Kilpikoski S (2010) The McKenzie method in assessing, classifying and treating non-specific

402 low back pain in adults with special reference to the centralization phenomenon. vol 158.

403 University of Jyväskylä,

404 3. Oliveira IOd, Pinto LLS, Oliveira MAd, Cêra M (2016) McKenzie method for low back pain.

405 Revista Dor 17 (4):303-306

406 4. Irandoust K, Taheri M, Mirmoezzi M, H'mida C, Chtourou H, Trabelsi K, Ammar A,

407 Nikolaidis PT, Rosemann T, Knechtle B (2019) The Effect of Aquatic Exercise on Postural

408 Mobility of Healthy Older Adults with Endomorphic Somatotype. International journal of

409 environmental research and public health $16(22): 4387$

410 5. Maher CG (2004) Effective physical treatment for chronic low back pain. Orthopedic Clinics

$41135(1): 57-64$

412 6. Jonsson E, Nachemson A (2000) Neck and back pain: the scientific evidence of causes,

413 diagnosis, and treatment. Lippincott Williams \& Wilkins,

414 7. Bello AI, Kalu NH, Adegoke BO, Agyepong-Badu S (2010) Hydrotherapy versus land-based

415 exercises in the management of chronic low back pain: A comparative study. Journal of

416 musculoskeletal research $13(04): 159-165$

417 8. Taheri M, Mirmoezzi M, Sabaghi M (2018) Effects of aquatic on balance and preventing of

418 fall among healthy elderly men. J Saf Promot Inj Prev 6:144-151

419 9. Machado LAC, De Souza MVS, Ferreira PH, Ferreira ML (2006) The McKenzie method for

420 low back pain: a systematic review of the literature with a meta-analysis approach. Spine 31

421 (9):E254-E262

422 10. Moldovan M (2012) Therapeutic Considerations and Recovery in Low Back Pain: Williams 
vs McKenzie. Timisoara Physical Education and Rehabilitation Journal 5 (9):58-64

424 11. Faul F, Erdfelder E, Lang A-G, Buchner A (2007) G* Power 3: A flexible statistical power

425 analysis program for the social, behavioral, and biomedical sciences. Behavior research methods

$426 \quad 39(2): 175-191$

427 12. Kobayashi D, Shimbo T, Hayashi H, Takahashi O (2019) Shiatsu for chronic lower back

428 pain: Randomized controlled study. Complementary therapies in medicine 45:33-37

429 13. Machado LA, Maher CG, Herbert RD, Clare H, McAuley JH (2010) The effectiveness of

430 the McKenzie method in addition to first-line care for acute low back pain: a randomized

431 controlled trial. BMC medicine $8(1): 10$

432 14. Association AE (2017) Aquatic fitness professional manual. Human Kinetics,

433 15. Mirmoezzi M, Yousefi M, Salmanpour M (2019) The Effects of Aquatic Isometric and

434 Isotonic Resistance Exercises on Fatigue Index of Aged Men. Sleep and Hypnosis (Online) 21

$435 \quad(1): 44-50$

436 16. Vergroesen P-P, Kingma I, Emanuel KS, Hoogendoorn RJ, Welting TJ, van Royen BJ, van

437 Dieën JH, Smit TH (2015) Mechanics and biology in intervertebral disc degeneration: a vicious

438 circle. Osteoarthritis and cartilage $23(7): 1057-1070$

439 17. Esther JO (2012) Therapeutic exercises in the management of non-specific low back pain.

$440 \quad$ Low back pain Croatia (EU): InTech:225-246

441 18. Hayden JA, Van Tulder MW, Malmivaara AV, Koes BW (2005) Meta-analysis: exercise

442 therapy for nonspecific low back pain. Annals of internal medicine 142 (9):765-775

443 19. Waller B, Lambeck J, Daly D (2009) Therapeutic aquatic exercise in the treatment of low

444 back pain: a systematic review. Clinical rehabilitation 23 (1):3-14

445 20. Stark MA, Rudell B, Haus G (2008) Observing position and movements in hydrotherapy: A

446 pilot study. Journal of Obstetric, Gynecologic \& Neonatal Nursing 37 (1):116-122

447 21. Sawant RS, Shinde SB (2019) Effect of hydrotherapy based exercises for chronic

448 nonspecific low back pain. Indian J Physiother Occup Ther 13 (1) 
449 22. Dundar U, Solak O, Yigit I, Evcik D, Kavuncu V (2009) Clinical effectiveness of aquatic

450 exercise to treat chronic low back pain: a randomized controlled trial. Spine 34 (14):1436-1440

451 23. Chiou S, Shih Y, Chou L, McGregor A, Strutton P (2014) Impaired neural drive in patients

452 with low back pain. European Journal of Pain 18 (6):794-802

453 24. Sousa CDDd, Nunes ACL, Jesus-Moraleida FRd (2017) Association between Physical

454 Activity and Disability in patients with low back pain. Motriz: Revista de Educação Física 23

$455 \quad(2)$

456 Sharma SS, Sheth MS (2018) Effect of neurodynamic mobilization on pain and function in

457 subjects with lumbo-sacral radiculopathy. Medicine Science 7:5-8

458

459

460

461

462

463

464

465

466

467

468

469

470

471

472

473

474

475

476

477

478 Table 1: Contents of the hydrotherapy program (main part) used in this study 


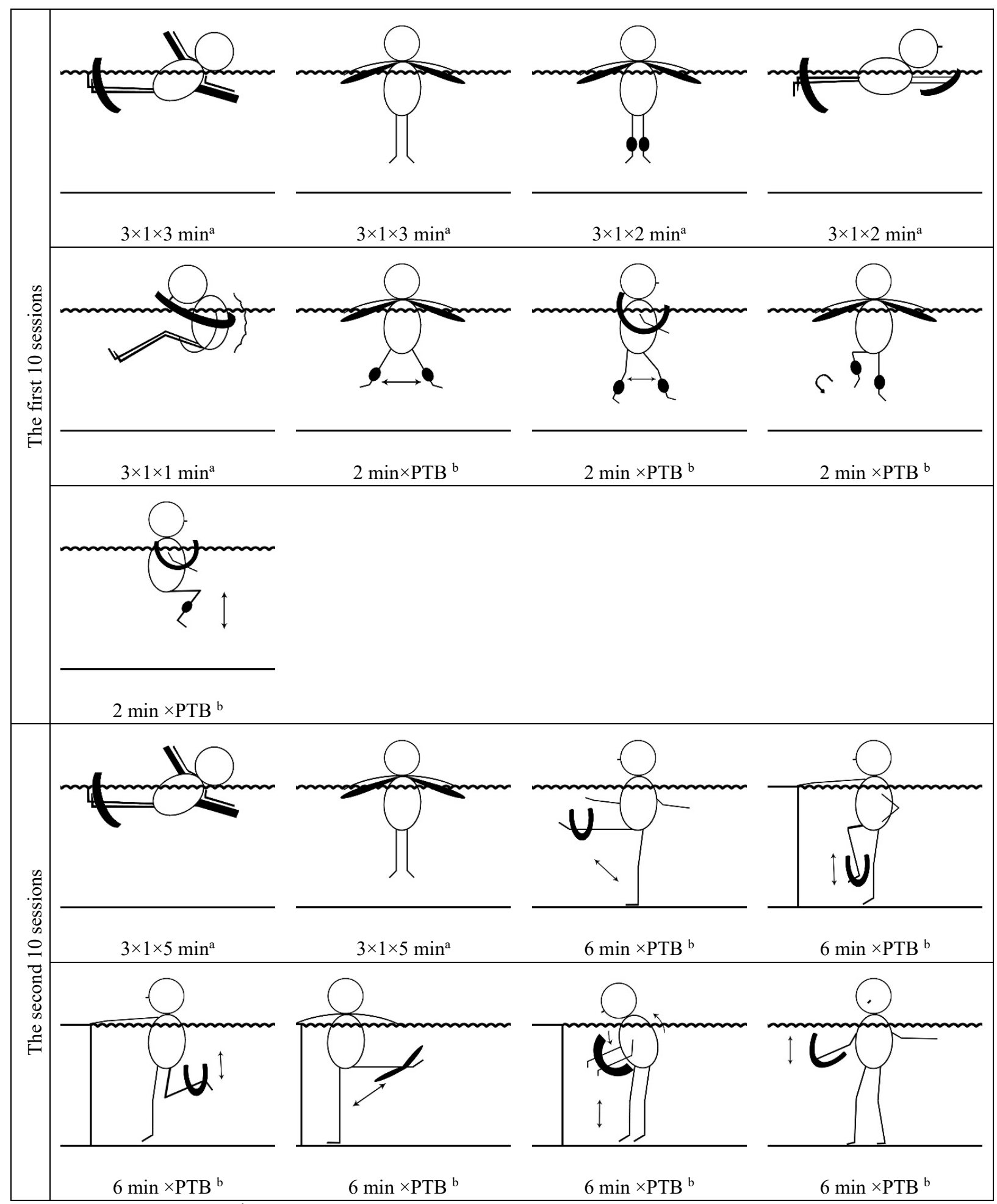

${ }^{a}$ Sets $\times$ Repetitions $\times$ Time. ${ }^{b}$ Time $\times$ PTB (to the Pain Thresholdand and Bilateral). Abbreviations: min: minutes; s: Seconds. The exercise sessions lasted about one hour. The rest between sets was 30 seconds. 
485 Table 2: Comparison of baseline characteristics between patients in hydrotherapy exercises and 486 control groups

\begin{tabular}{llll}
\hline & Hydrotherapy $(\mathrm{n}=14)$ & Control $(\mathrm{n}=14)$ & $\mathrm{p}$-value \\
\hline \multicolumn{1}{c}{ Demographics } & & & \\
Age, mean (SD) & $42.99(4.55)$ & $39.12(6.12)$ & 0.068 \\
Gender & Male:9 (64.29) & Male 7 (50) & 0.071 \\
& Female: 5(35.71) & Female: 7 (50) & 0.364 \\
Height, mean, cm (SD) & $172.22(6.64)$ & $174.41(5.87)$ & 0.370 \\
Weight, mean, kg (SD) & $71.11(7.54)$ & $73.44(5.90)$ & 0.759 \\
Body mass index, mean, kg/m2 (SD) & $23.99(1.84)$ & $24.22(2.08)$ & 0.251 \\
$\quad$ & & & 0.644 \\
Mumeric Pain Rating Scale, mean (SD) & $6.57(1.02)$ & $5.9(1.77)$ & 0.527 \\
Roland-Morris Disability Questionnaire, mean (SD) & $10.9(5.52)$ & $10.75(5.83)$ & $39.4(15.1)$ \\
Straight Leg Test, mean, degree (SD) & $38.8(14.6)$ & & \\
\hline
\end{tabular}


489 Table 3: Comparison on the mean change in the duration of befor $1^{\text {st }}$ season (baseline), $10^{\text {st }}$ season and $20^{\text {st }}$ season

\begin{tabular}{|c|c|c|c|c|c|}
\hline Outcome variables & $\begin{array}{c}\text { Baseline } \\
\text { Mean } \pm \text { S.D. }\end{array}$ & $\begin{array}{c}10^{\text {st }} \text { season } \\
\text { Mean } \pm \text { S.D. }{ }^{\text {a }}\end{array}$ & $\begin{array}{c}20^{\text {st }} \text { season } \\
\text { Mean } \pm \text { S.D. }{ }^{\text {a }}\end{array}$ & p-value & $\begin{array}{c}\text { Effect } \\
\text { size }^{\text {b }}\end{array}$ \\
\hline (1) NPRS & & & & $0.001^{\mathrm{c}}$ & \\
\hline Hydrotherapy group & $6.57 \pm 1.02$ & $3.79 \pm 1.37$ & $3.43 \pm 1.55$ & $<0.001^{\mathrm{d}}$ & 0.815 \\
\hline Control group & $5.90 \pm 1.77$ & $5.64 \pm 1.28$ & $5.57 \pm 0.85$ & $0.377^{\mathrm{d}}$ & \\
\hline (2) RMDQ & & & & $<0.001^{\mathrm{c}}$ & \\
\hline Hydrotherapy group & $10.9 \pm 5.52$ & $6.8 \pm 4.85$ & $6.52 \pm 4.51$ & $<0.001^{\mathrm{d}}$ & 0.541 \\
\hline Control group & $10.75 \pm 5.83$ & $10.13 \pm 5.9$ & $10.2 \pm 5.66$ & $0.544^{\mathrm{d}}$ & \\
\hline (3) SLRT & & & & $0.015^{\mathrm{e}}$ & \\
\hline Hydrotherapy group & $38.8 \pm 14.6$ & $85.4 \pm 12.1$ & $87.1 \pm 10.9$ & $<0.001^{\mathrm{f}}$ & 0.717 \\
\hline Control group & $39.4 \pm 15.1$ & $41.7 \pm 14.4$ & $43.6 \pm 16.2$ & $0.671^{\mathrm{f}}$ & \\
\hline
\end{tabular}

${ }^{\mathrm{a}}$ S.D. $=$ standard deviation of the mean.

$492{ }^{\mathrm{b}}$ Effect size is calculated by Kendall's W=X2/N(K-1).

$493{ }^{\mathrm{c}} \mathrm{p}$-value is calculated by Mann-Whitney U statistics for between-groups comparison.

$494{ }^{\mathrm{d}} \mathrm{p}$-value is calculated by Friedman statistics for within-group comparison.

$495{ }^{\mathrm{e}} \mathrm{p}$-value is calculated by Two-way repeated measure ANOVA between-groups comparison.

${ }^{\mathrm{f}} \mathrm{p}$-value is calculated by Two-way repeated measure ANOVA for within-group comparison 


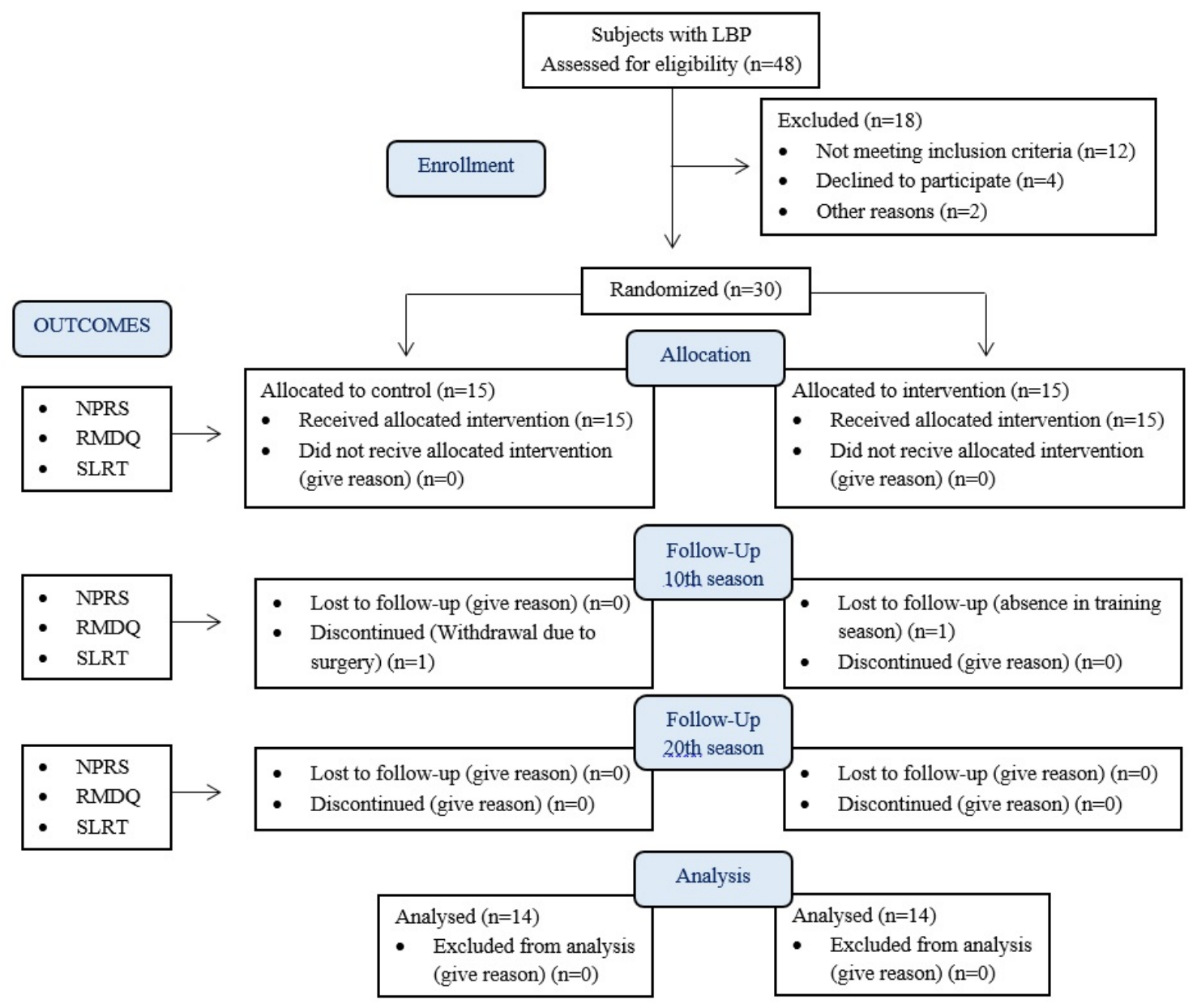

Figure 1: CONSORT flow chart indicating flow of subjects through the trial. Abbreviation: NPRS, 503 Numeric Pain Rating Scale; RMDQ, Roland-Morris Disability Questionnaire; SLRT, Straight Leg 


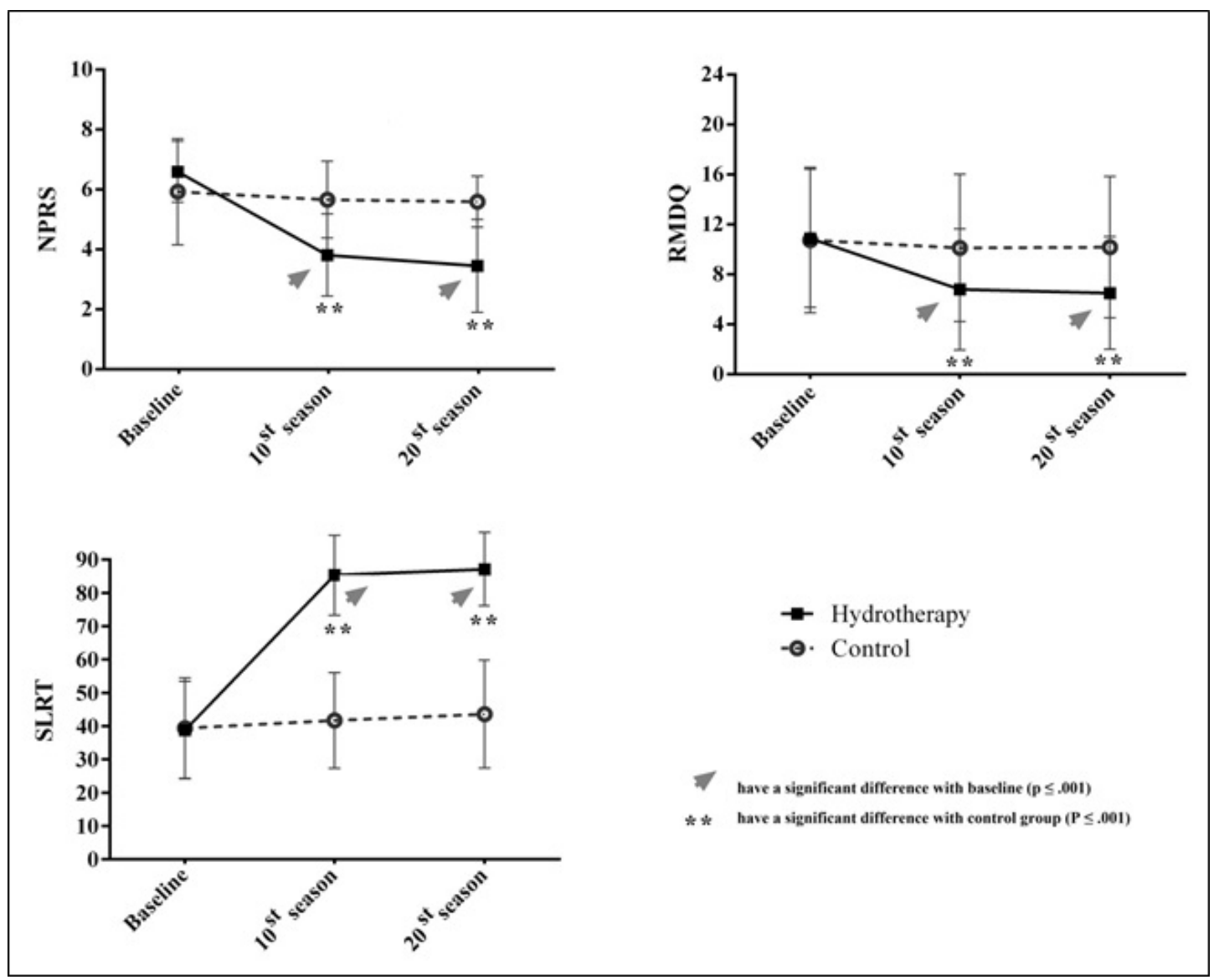

516 Figure 2: Comparison on the mean change in the duration between groups.

517

518

519

520

521

522

523 